\title{
APPLICATIONS OF DUALITY
}

\section{EBEN MATLIS}

Let $R$ and $S$ be two rings with units; then Cartan and Eilenberg have established the following duality identities (1) and (2) [3, Chapter 5, §5]. In the situation described by the symbol $\left({ }_{R} A,{ }_{S} B_{R},{ }_{S} C\right)$, where $C$ is $S$-injective, we have an identity:

$$
\operatorname{Ext}_{R}\left(A, \operatorname{Hom}_{S}(B, C)\right) \cong \operatorname{Hom}_{S}\left(\operatorname{Tor}^{R}(B, A), C\right) .
$$

In the situation described by the symbol $\left({ }_{R} A,{ }_{R} B_{S}, C_{S}\right)$, if $R$ is left Noetherian, $A$ is finitely $R$-generated, and $C$ is $S$-injective, then we have a second identity:

$$
\operatorname{Hom}_{S}\left(\operatorname{Ext}_{R}(A, B), C\right) \cong \operatorname{Tor}^{R}\left(\operatorname{Hom}_{S}(B, C), A\right) .
$$

In this note we shall apply these identities to the solution of two entirely dissimilar problems. First we shall prove that if $R$ is a left Noetherian ring, then f.l.inj. $\operatorname{dim} R=$ f.r.w. $\operatorname{dim} R$. Secondly, we shall give a new, short proof of a theorem of Kaplansky [5] which states that a ring $R$ is a Prufer ring if and only if, the torsion submodule of any finitely generated $R$-module is a direct summand.

Definition. Let $R$ be a ring with unit. If $A$ is a left $R$-module, we shall denote the injective dimension of $A$ as an $R$-module by inj. $\operatorname{dim}_{R} A$. If $B$ is a right $R$-module, we shall denote the weak dimension of $B$ as an $R$-module by w. $\operatorname{dim}_{R} B$. We then define the finitistic, left, injective, dimension of $R$ by f.l.inj. $\operatorname{dim} R=$ sup. inj. $\operatorname{dim}_{R} A$, where $A$ ranges over all left $R$-modules of finite injective dimension. Similarly, we define the finitistic, right, weak dimension of $R$ by f.r.w. $\operatorname{dim} R=$ sup.w. $\operatorname{dim}_{R} B$, where $B$ ranges over all right $R$-modules of finite weak dimension.

Lemma 1. Let $A$ be a left module over a ring $R$. Then inj. $\operatorname{dim}_{R} A \leqq n$ if and only if, $\operatorname{Ext}_{R}^{n+1}(R / I, A)=0$ for every left ideal $I$ of $R$.

Proof. If inj. $\operatorname{dim}_{R} A \leqq n$, then of course $\operatorname{Ext}_{R}^{n+1}(X, A)=0$ for all left $R$-modules $X$. Conversely, suppose that $\operatorname{Ext}_{R}^{n+1}(R / I, A)=0$ for all left ideals $I$ of $R$. Let

$$
0 \rightarrow A \rightarrow C_{0} \rightarrow C_{1} \rightarrow \cdots \rightarrow C_{n-1} \rightarrow D \rightarrow 0
$$

be an exact sequence of left $R$-modules, where $C_{i}$ is $R$-injective for $i=0, \cdots, n-1$. Then $0=\operatorname{Ext}_{R}^{n+1}(R / I, A) \cong \operatorname{Ext}_{R}^{1}(R / I, D)$ for all left

Received by the editors November 12, 1958. 
ideals $I$ of $R$. Hence $D$ is $R$-injective [3, Proposition 1.3.2], and thus inj. $\operatorname{dim}_{R} A \leqq n$.

Lemma 1 provides an easy proof of the following theorem of $\mathrm{M}$. Auslander [1, Theorem 1]. (It has been brought to my attention that this proof was known, but unpublished, by S. Eilenberg).

COROLlary. For any ring $R$, l.gl. $\operatorname{dim} R=$ sup. $h d_{R} R / I$, where $I$ ranges over the left ideals of $R$.

Proof. If sup. $h d_{R} R / I=\infty$, then 1.gl. $\operatorname{dim} R=\infty$. Hence assume that sup. $h d_{R} R / I=n$. Then $\operatorname{Ext}_{R}^{n+1}(R / I, A)=0$ for all left $R$-modules $A$ and all left ideals $I$ of $R$. Thus by Lemma $1 \mathrm{inj}$. $\operatorname{dim}_{R} A \leqq n$ for all left $R$-modules $A$, and thus l.gl. $\operatorname{dim} R \leqq n$.

The following lemma is well known, but we will prove it for the sake of completeness.

Lemma 2. Let $B$ be a right module over a ring $R$. Then $w \cdot \operatorname{dim}_{R} B \leqq n$ if and only if, $\operatorname{Tor}_{n+1}(B, R / I)=0$ for every left ideal $I$ of $R$.

Proof. If w. $\operatorname{dim}_{R} B \leqq n$, then by definition $\operatorname{Tor}_{n+1}^{R}(B, X)=0$ for every left $R$-module $X$. Conversely, assume that $\operatorname{Tor}_{n+1}^{R}(B, R / I)=0$ for every left ideal $I$ of $R$. We assume by induction that $\operatorname{Tor}_{n+1}^{R}(B, Y)$ $=0$ for every left $R$-module $Y$ generated by fewer than $m$ elements. Let $E$ be a left $R$-module generated by $m$ elements, and $E_{1}$ a submodule of $E$ generated by one of these elements. Then $E / E_{1}$ is generated by fewer than $m$ elements. Hence from the exact sequence:

$$
0 \rightarrow E_{1} \rightarrow E \rightarrow E / E_{1} \rightarrow 0
$$

we obtain the exact sequence:

$$
0=\operatorname{Tor}_{n+1}^{R}\left(B, E_{1}\right) \rightarrow \operatorname{Tor}_{n+1}^{R}(B, E) \rightarrow \operatorname{Tor}_{n+1}^{R}\left(B, E / E_{1}\right)=0 .
$$

Thus $\operatorname{Tor}_{n+1}^{R}(B, E)=0$ for all finitely generated left $R$-modules $E$. Since Tor commutes with direct limits, $\operatorname{Tor}_{n+1}^{R}(B, X)=0$ for all left $R$-modules $X$. Thus w. $\operatorname{dim}_{R} B \leqq n$.

Definition. Let $Z$ denote the integers, and $K$ the rationals (mod one). If $X$ is a left (or right) module over a ring $R$, then $X^{*}$ $=\operatorname{Hom}_{Z}(X, K)$ is a right (or left) module over $R$. It is easily seen that $X=0$, if and only if $X^{*}=0$.

Lemma 3. Let $R$ be any ring. Then f.r.w. $\operatorname{dim} R \leqq$ f.l.inj. $\operatorname{dim} R$.

Proof. Let $B$ be any right $R$-module, and $A$ any left $R$-module. Then by (1) $\operatorname{Ext}_{R}\left(A, B^{*}\right) \cong \operatorname{Tor}^{R}(B, A)^{*}$. Thus w. $\operatorname{dim}_{R} B$ 
$=$ inj. $\operatorname{dim}_{R} B^{*}$. It follows immediately that f.r.w. $\operatorname{dim} R$ $\leqq$ f.l.inj. $\operatorname{dim} R$.

Theorem 1. Let $R$ be a left Noetherian ring. Then f.l.inj. $\operatorname{dim} R$ =f.r.w. $\operatorname{dim} R$.

Proof. Let $I$ be a left ideal of $R$, and $A$ a left $R$-module. Then by (2) $\operatorname{Ext}_{R}(R / I, A)^{*} \cong \operatorname{Tor}^{R}\left(A^{*}, R / I\right)$. Hence by Lemmas 1 and 2 , inj. $\operatorname{dim}_{R} A=$ w. $\operatorname{dim}_{R} A^{*}$. Thus f.l.inj. $\operatorname{dim} R \leqq$ f.r.w. $\operatorname{dim} R$. The opposite inequality is provided by Lemma 3 .

Of course if $R$ is a commutative, Noetherian ring, then there is no distinction between left and right modules; and then f.inj. $\operatorname{dim} R$ $=$ f.w. $\operatorname{dim} R$. It then follows from [2, Theorem 2.4] that f.inj. $\operatorname{dim} R$ $\leqq$ Krull $\operatorname{dim} R$.

As a final application of duality we give a new proof of a theorem of Kaplansky [5].

Definition. A Prufer ring is an integral domain in which every finitely generated ideal is invertible.

Theorem 2. Let $R$ be an integral domain. Then $R$ is a Prufer ring if and only if the torsion submodule of every finitely generated $R$-module is a direct summand.

Proof. If $R$ is a Prufer ring, a finitely generated, torsion-free $R$ module is projective, and the condition follows. Conversely, assume that the torsion submodule of every finitely generated $R$-module is a direct summand. Let $S$ be a finitely generated, torsion-free $R$-module, and $I$ an ideal of $R$. By [4, Theorem 2] and Lemma 2 it is sufficient to prove that $\operatorname{Tor}_{1}^{R}(S, R / I)=0$.

Suppose that $\operatorname{Tor}_{1}^{R}(S, R / I) \neq 0$. Since $\operatorname{Tor}_{1}^{R}(S, R / I)$ is a torsion module, there exists a torsion, injective $R$-module $C$ such that $\operatorname{Hom}_{R}\left(\operatorname{Tor}_{1}^{R}(S, R / I), C\right) \neq 0$. By (1) we have $\operatorname{Ext}_{R}^{1}\left(S, \operatorname{Hom}_{R}(R / I, C)\right)$ $\cong \operatorname{Hom}_{R}\left(\operatorname{Tor}_{1}^{R}(S, R / I), C\right)$. Since $\operatorname{Hom}_{R}(R / I, C)$ is a torsion module, to establish a contradiction it will be sufficient to prove that $\operatorname{Ext}_{R}^{1}(S, R)=0$ for any torsion module $T$.

Let $A$ be an extension of $T$ by $S$. Now $S$ is generated by a finite number of elements $\left\{y_{1}, \cdots, y_{n}\right\}$. Choose $x_{i} \in A$ such that $x_{i} \rightarrow y_{i}$, and let $B=\left(x_{1}, \cdots, x_{n}\right) \subset A$. Then $T \cap B$ is the torsion submodule of $B$. Hence by assumption $B=(T \cap B) \oplus S^{\prime}$, where $S^{\prime} \cong S$. Then $A=T \oplus S^{\prime}$, and thus $\operatorname{Ext}_{R}^{1}(S, T)=0$.

\section{BiBLIOGRAPHY}

1. M. Auslander, On the dimension of modules and algebras (III), Nagoya Math. J. vol. 9 (1955) pp. 67-77. 
2. M. Auslander and D. Buchsbaum, Homological dimension in Noetherian rings, II, Trans. Amer. Math. Soc. vol. 88 (1958) pp. 194-206.

3. H. Cartan and S. Eilenberg, Homological algebra, Princeton University Press, 1956.

4. A. Hattori, On Prufer rings, J. Math. Soc. Japan vol. 9 no. 4 (1957) pp. 381385.

5. I. Kaplansky, A characterization of Prufer rings, J. Indian Math. Soc. (forthcoming).

NORTHWESTERN UNIVERSITY 